\title{
Methods and methodologies in Second language writing research
}

\author{
Ken Hyland \\ University of Hong Kong
}

\begin{abstract}
Published as: Hyland, K. (2016). Methods and methodologies in second language writing. System. Vol 59: 116-125.
\end{abstract}

\begin{abstract}
A considerable variety of methods have been used to understand the complex, multifaceted nature of L2 writing. Often driven by pedagogical imperatives and informed by particular views of writing, texts and writers, these methods themselves raise questions regarding what we believe writing is and about our interpretive practices. With increasing numbers of teachers and scholars turning to investigate writing in their classrooms or courses of further study, it may be helpful to be aware of what options are available for studying writing and how these relate to key methodological designs. More than this, however, it is important to be aware of what our choices imply about our understanding of what writing is and how it can be known. In this paper I set out the main approaches to studying writing for novice researchers, providing examples of key studies, and go on to situate these methods within the main theories about writing arguing that methods are not neutral options but allow us to see certain things but not others. They do not just tell us different things about writing but reveal what we believe writing to be.
\end{abstract}




\section{Methods and methodologies in Second language writing research}

\section{Introduction}

Over the last two decades, second language writing has evolved into a well-established field of inquiry characterized by defined areas of interest, distinct methods of inquiry, and networks of conferences, journals and professional organizations for the dissemination of knowledge among practitioners. Teachers have come to recognize the value of research. Once seen as a distinct scholarly activity divorced from the hard realities of the classroom, the emergence of more contextsensitive pedagogies encourages us to better understand the texts we present in our classes, the ways our students write, and how target communities use the texts that are important to them. Teachers of writing are, then, increasingly becoming researchers of writing, developing an understanding of texts and establishing a basis for reflection which in turn feeds back into, and improves, teaching.

The kind of systematic research-reflection cycle mentioned above is termed action research (e.g. Burns, 2013) and it has clearly done a lot to democratize research by putting it into the hands of teachers, and to professionalize teachers by giving them new skills and knowledge to bring to their classrooms. But research just as often originates in a desire to satisfy curiosity than solve problems. When talking of case studies, for example, Stake (2005), distinguishes between intrinsic and instrumental types, the former undertaken because of its interesting particularity and ordinariness, rather than of its benefits to others or contributions to a literature. It is useful, therefore, for novice researchers to see what their options are when selecting inquiry methods and it is equally important for them to understand what these choices imply about writing, students and learning. In this paper I want to offer some guidelines for novice researchers in second language writing research and to argue that research methods and designs are not neutral options but are motivated and allow us to see certain things but not others. First, I begin with a brief review of the key approaches to researching writing then discuss how these methods relate to wider epistemological perspectives. 


\section{Methods and methodologies in L2 writing research}

Research generally begins by isolating something that interests or worries us and then asking questions about it. The kinds of questions we ask, however, and how we collect, analyze and interpret the data to answer them, depend on our preferences and preconceptions, the topic and the purpose we have for studying it, the context, our access to data, the time and resources we have, and the energy we are prepared to invest. There is no 'one-size-fits-all' formula to carrying out research on writing (or on anything else) but nor is there a perfect approach to every question. While research can answer questions that interest us, there is rarely only one answer or one 'truth' waiting to be revealed. In fact, almost any research design can answer any research question. But while the research approach we adopt will tell us something about the thing we are studying, it is important to be aware of the assumptions we are making when we design our research and select our tools for collecting data.

Here we need to distinguish between methods, or ways of collecting data (such as observations, surveys and interviews), and methodologies, the principles and understandings that guide and influence our choice and use of methods (like experimentation and ethnography). Methods are the front line techniques and methodologies the systematic application of them.

Methodology, therefore, concerns how research is done, how we find out about things, and how knowledge is gained. While it clarifies, explains and justifies the choice of certain methods in our research, researchers (and journals) often tend to favour some methodologies over others and regard those as uniquely legitimate or effective. In fact, the choice of methodology we adopt to study L2 writing will largely depend on what we believe writing is, the model of language we subscribe to, and how we understand learning. Methodology, then, is a general strategy or operating model for conducting research: a plan which contains a logical organization and the directions to answer a research question. It shapes how methods are used but does not determine the data required, how these data are to be collected or how they should be analyzed. Thus collecting naturally-produced student texts, a method, might be done as part of a wider ethnographical study, as part of a controlled experiment to compare groups of writers, or as data for studying learner improvement over time (methodologies). 


\section{Research methods}

Because method is often used to refer to all research processes, it is helpful to clarify the differences so that teachers can see the research options available to them. First, there are four broad ways of collecting data related to writing and these are set out in Table 1 and elaborated briefly below.

Table 1: Major methods used in researching writing

Elicitation: $\quad$ Ways of prompting self-report and performance data

Introspection: Ways of collecting verbal or written reports by text users

Observation: Direct or recorded data of 'live' interactions or writing behaviour

Text samples: Collections of naturally produced samples of writing

3.1 Elicitation refers to methods for prompting self-report and performance data.

- Questionnaires are useful for collecting large amounts of structured, easily analysable information about text users' characteristics, beliefs or attitudes, information that is not usually available from observation of their behaviour or from their texts. Like interviews, they allow researchers to tap people's views and experiences of writing, but are more quantitative and restrictive. They have been widely used in writing research to discover the kinds of writing target communities require. Evans and Green (2007), for example, used a questionnaire to survey 5,000 Hong Kong students about the difficulties they experience when studying through the medium of English, identifying problems of style, grammar and cohesion.

- Interviews offer more interactive and less predetermined ways of eliciting information than surveys and so allow greater flexibility and potential for elaboration. Although sometimes simply oral questionnaires, highly structured and limiting responses, interviews generally represent a very different way of understanding human experience. Semi-structured formats, which loosely follow a set of guidelines and allow extensive follow-up, or unstructured types, which observe an outline of issues but follow the direction of interviewee responses, regard knowledge as generated between people rather than as objectified and external to them. Participants are able to discuss their interpretations and perspectives, sharing what writing means to them rather than responding to 
preconceived categories. This flexibility and responsiveness means that interviews are used widely in writing research to learn more about attitudes to writing, about teaching and learning and about reasons for rhetorical choices. They are therefore helpful in learning about how writers understand what it is they do when they write and are particularly valuable in revealing issues that might be difficult to predict, such as how students interpret teacher written feedback (e.g. Author, 2013).

- Focus groups are more interactive and less threatening than interviews as participants are free to talk with other group members. They therefore take some control away from the interviewer, but can produce richer data as a result, although what participants tell the researcher is shared with other group participants as well, raising privacy concerns and limiting the kinds of topics that the researcher can pursue. Usually conducted face-to-face, they may also be held in synchronous computer-mediated venues such as skype or on chatrooms where transcripts can be saved and considered later. Groups have been used to discover students' academic writing needs and difficulties (e.g. Zhu \& Flaitz, 2005).

- Tests, or one-shot writing tasks, elicit performance information from students, discovering what it is they know, can do or are able to remember in writing. They therefore offer insights into students' writing ability and knowledge of genre, language forms and rhetorical understandings and, indirectly, can also provide information about rater behaviour and judgements of good writing. Weigle (1999), for example, investigated how experienced and inexperienced raters scored essays written by ESL writers on two different prompts, finding that inexperienced raters were more severe on one prompt but that differences between the two groups were eliminated following rater training.

\subsection{Introspection}

The use of verbal reports as data reflects the idea that the process of writing requires conscious attention and that at least some of the thought processes involved can be recovered, either by talking aloud while writing or as retrospective recalls.

- Think aloud protocols (TAPs) involve participants writing in their normal way but instructed to verbalise what they are doing at the same time, so that information can be recorded on their decisions, 
strategies and perceptions as they work. This kind of data has been criticised as offering an artificial and incomplete picture of the complex cognitive activities involved in writing as many such processes are routine and not available to verbal description while the act of reporting may create reactive effects, distorting the process being reported on. Yang, Hu and Wang (2014), however, found verbalization had little impact on students' writing in controlled conditions. In the absence of alternatives, the method has been widely used to reveal the strategies writers use when composing, particularly what students do when planning and revising (e.g. Wong, 2005). Stimulated recalls, on the other hand, involve videotaping the writer while writing then discussing the writer's thought processes while watching the video together immediately afterwards. This is the approach taken by Lei (2008) when studying student writing strategies.

- Diaries are first-person entries in a journal or blog and then analysed for recurring patterns or significant events. Diarists can be asked to produce 'narrative' entries which freely introspect on their learning or writing experiences, or follow guidelines to restrict the issues addressed. These can be detailed points to note (who is your reader for this essay?) or a loose framework for response (note all the work you did to complete this task). Alternatively, researchers may ask diarists to concentrate only on 'critical incidents' of personal significance or to simply record dates and times of writing and what it was they wrote. While some diarists may resent the time and intrusion this involves, diaries provide a rich source of reflective data which can reveal processes difficult to access in other ways. Thus Li (2007) used a Chinese scholar's blog entries to discover how he went about writing a paper in English for publication.

\subsection{Observation}

While elicitation and introspective methods report what people say they think and do, observations give evidence of it. They are based on conscious noticing and precise recording of actions as a way of seeing these actions in a new light.

- Recording behaviour can be done live or from taped recordings. Again the researcher can impose different degrees of structure on the mass of data that this method can often produce, from 
simply checking pre-defined boxes at fixed intervals or every time a type of behaviour occurs, to writing a full narrative of events. The most structured observations involve a prior coding scheme to highlight significant events but one problem here is that all observations privilege some behaviours and neglect others, as we only record what we think is important. Ticking pre-selected actions gives more manageable data, but it may ignore unexpected behaviour. This explains why the method is often combined with others, as in Louhiala-Salminen's (2002) study of a Business Manager's activities through one day. In addition to observation, she recorded oral encounters, analysed copies of written materials and conducted interviews to gain as full a picture as possible.

- Keystroke logging is a more reliable, and less intrusive, way of tracking writing behavior than a researcher looking over a writer's shoulder as software such as Inputlog or Scriptlog can record and playback what writers do as they write. This logs and time stamps keystrokes, pauses, cutting, pasting, and deleting and mouse activity, allowing the researcher to reconstruct text production processes and sites visited. Closely associated with psycholinguistic aspects of writing, the method can show how a writer juggles the complexities of cognitive activities in real time, planning content, choosing forms, considering audience and style, and so on. Leijten and Van Waes (2013), for example, reveal how a group of Master's students and experts used sources when writing short texts, describing the organization of writing processes that involve searching, reading, and copying from multiple digital sources.

\subsection{Text data}

A major source of data is writing itself: the use of texts as objects of study. Approaches which analyse texts see writing as an outcome of activity, as words on a page or screen and can be descriptive (revealing what occurs), analytical (interpreting why it occurs) or critical (questioning the social relations which underlie and are reproduced by what occurs). Because they can be approached in different ways for different purposes text analyses can be seen as both methodology and method as 
researchers seek to understand what language choices writers make, why they make them and what they mean. I will return to analyses below, but here I focus on data collection.

- Single text Some studies focus on a single text as an instance in action, either because the text seems interesting or represents a particular genre or author, such as Martin's (2004) analysis of the ways a writer negotiates solidarity with his readers in a Hong Kong lifestyle magazine.

- chain of texts Another source of text data is the collection of a series of texts gathered according to some principle of organisation. This may be to observe the changes made by a single writer over several drafts, such as Li's (2006) account of the transformations over six drafts of a research paper by a Chinese scholar. Alternatively, chains can be observed to see how different purposes, audiences and genres influence rhetorical choices. Brown's (2011) analysis of revisions made in parallel passages from introductions in six humanities and social science dissertation-monograph pairs, for example, highlights the revisions transforming a $\mathrm{PhD}$ dissertation into a book. Researchers have also explored historical changes in a single genre over time (Author, 2016).

Corpora Perhaps most commonly now, analysts study collections of texts, to get a more representative picture of a genre or a group of writers. A corpus represents a writer's experience of language in some restricted domain, providing an alternative to intuition, initially looking at the frequency with which words or patterns occur and how features commonly associate in collocational patterns, pointing to common usage in the genre.

\section{Methodologies}

While these sources of data and the methods used to collect them are perhaps familiar to many teachers, methodologies are often less visible. I now turn to some of the major methodological designs, summarised in Table 2. 
Table 2: Major design methodologies in L2 writing research

Experimentation: Controlled context to discover the effect of an intervention

Ethnography: Prolonged engagement with a community to capture participant perspectives

Auto-ethnography: author reflects on personal experience and social meaning of writing

Critical analysis: Study connections between situated writing and wider socio-political contexts

Discourse analysis: Study of authentic examples of writing used for communication in natural settings

Meta-analyses: An empirical synthesis to provide an overview of results of studies in an area

\subsection{Experimentation}

Experimental methods are set up to discover the effect of something on something else.

Experimentation is a deductive method in that the researcher intervenes to test a theory by isolating and studying a single feature under controlled conditions. Most simply, this involves applying some treatment to one of two groups while holding other factors constant. The two groups are then given a post test to see the effect of the treatment and statistical tests are carried out to find out if differences between the control and the experimental groups are significant. More complex treatments such as factorial designs can be used to explore several treatment variables at several levels of interactions (McGowan, 2011). Experimental researchers are particularly concerned with internal validity, or whether changes are only due to the issue under study, and external validity, whether the results are generalizable to other populations. The use of test measurements also has to be carefully monitored to minimize threats to the reliability and validity of the research.

Writing research, however, largely follows a 'relativist' orientation and favours more qualitative, natural, and 'thicker' data collection techniques. But although experimental research can never capture the rich complexity of writing, the method has been used to look at the impact of instruction or feedback on writing. In one interesting experiment on peer review, for instance, Lundstrom and Baker (2009) divided students into those who reviewed anonymous papers but received no peer feedback and those who received feedback but did not review other students' writing. An analysis of students' writing over a semester showed that the givers made more significant gains in their own 
writing than did the receivers, especially at lower proficiency levels. Experimental studies should be treated cautiously, however, as it is difficult to hold all non-experimental variables constant in natural settings so differences in teaching styles, learner preferences, teacher attitudes, peer relationships, and so on can all influence results.

4.2 Ethnography seeks to provide a rich (or 'thick') description and interpretive account of what people do in a classroom, workplace or other social setting, the outcome of their interactions in that setting and how they understand what they are doing. Researchers use a range of diverse methods, particularly observation and elicitation, to understand behaviour from participants' perspectives. As far as possible, they attempt to collect and analyse data over a period without pre-set categories or explicit hypotheses. Because it uses multiple methods and involves sustained engagement with a context it is labour-intensive and time-consuming, demanding considerable know-how and resources (Hesse-Biber \& Leavy, 2006). While sometimes criticised for a lack of generalizability, ethnography allows for a detailed explanation of what is specific to a particular group.

Researchers generally study language practices within familiar communities and institutions rather than in far flung exotic locations, as in anthropological ethnographies. Language is always considered within the context of its production and reception, rather than in isolation, simply as text. An approach to studying writing which highlights text analysis within more traditional ethnographic techniques, such as informal interviews and observations, is textography (Starfield et al, 2014). This aims to provide a more contextualized basis for understanding writing in the social, cultural and institutional settings in which it takes place than might be obtained by looking only at texts themselves. An example is Paltridge's (2008) study of the exegeses that art and design students write in their masters degrees, interviewing students, supervisors and examiners and studying their texts.

4.3 Auto-ethnography focuses on the researcher-writer's subjective experience rather than the beliefs and practices of others, connecting this to wider cultural and social meanings (Maréchal, 2010). Potentially this might provoke insight into often overlooked problems, such as the nature of identity, 
race, sexuality, life in academia, etc. Involving recall or diary methods, auto-ethnography seeks to assist the researcher make sense of his or her individual experience. They are, however, are also critical or even political as they often challenge us to consider things, or do them differently.

Chamcharatsri (2009) found auto-ethnography to be a valuable tool for L2 learners in a writing class, allowing them to explore and reflect on their cultural background and identities in a US university.

4.4 Critical Analysis is a methodology employing a range of methods to "identify linkages, broadly construed, between local occasions of language learning and use to broader social processes, formations and discourses" (Talmy, 2015: 153). Essentially the prefix critical can be added to other methodologies such as discourse analysis or ethnography to empirically explore these linkages. In writing research, methods such as text analysis, observations and interviews have been used widely. All three methods, for example, were used by Liu and Tannacito (2013) in their study of two Taiwanese students exhibiting agency and identity investment through resistance to the practices of an American writing classroom, with the authors relating their actions to ideological implications about ethnicity, race, and class.

\subsection{Text Analysis}

Texts can be approached in different ways and for different purposes: looking at systems of choices, institutional ideologies, L1 and L2 practices, what they say about communities of users and how they link to other texts. Broadly, texts can be understood in two ways. First, they can be looked at as systems of forms, focusing on grammatical items or patterns to better understand the regularities we find in texts or student errors. Second, and more usually, texts are treated as discourse, or how they work to communicate in particular settings. Here texts are resources to accomplish writer goals and understood as language in action, generally focusing on particular genres, such as a newspaper editorial, business report or argumentative essay.

Central to discourse analysis is the idea that forms express functions and this also underpins the highly productive notion of genre. Genres are abstract, socially recognized ways of using language and represent how writers typically respond to recurring situations. The ways people write are often 
not a matter of conscious awareness, but of routine and habit, acquired and changed through repeated interactions. Evidence for this routine behavior is often sought in specialized corpora using concordance programmes which bring together all instances of a search word or phrase in the corpus as a list of unconnected lines of text, showing instances of actual language $u s e$ when read horizontally but regularities of system when read vertically. Corpora can be analyzed using corpus-based or corpus-driven procedures (Tognini-Bonelli, 2001). The former is where the researcher begins with a pre-selected list of potentially productive items and examines the corpus for their frequencies and behaviour, such as Xiao et al's (2006) study of passive voice. Less common in writing studies are corpus-driven methods where the corpus is treated as the basis for any discovery, as in Biber et al's (2004) research to identify the most common multi-word patterns in textbooks.

\subsection{Meta-analyses}

This is an empirical synthesis or systematic review which aims to provide an exhaustive summary of current literature relevant to a research question. While it is customary to distinguish between research which is primary (uses new data) and secondary (reviews existing studies), meta-analyses differ from traditional literature reviews by adopting an empirical perspective, being as thorough as possible, and not taking the claims of report authors at face value. It uses an explicit approach and aims to identify what can reliably be said on the basis of these studies and the criteria used to evaluate them. They therefore go beyond the individual results contained in the original studies to produce synthetic findings. Norris and Ortega (2006) have published a collection which exemplifies the methodology for conducting synthesis research in applied linguistics and a good example of a study in writing is Truscott's (2007) review of error correction studies which shows teacher feedback on errors has a negative effect on learners' accuracy.

\subsection{Case studies}

A case-study is 'an instance in action'; a means of portraying what a particular situation is like by capturing the close-up reality of participants' lived experiences and thoughts about a situation. They typically combine methods to explore a particular bounded phenomena. The purpose of most case 
studies is to gain a better understanding of a person, process, group or context rather than to statistically generalise to other populations, although they do allow theoretical generalisations which can be valuable in other research contexts (Yin, 2014). Essentially, case studies blend a rich description of events with interpretive analysis that draws on participants' own perspectives. Such a thorough portrayal of local writing behaviours characterises a great deal of L2 writing research. One example is Li and Casanave's (2012) study of how two first year Hong Kong undergraduates understood plagiarism and their strategies based on these understandings in writing the same sourcebased assignment. The study drew on textual comparisons between student texts and source texts, interview data, and observation notes. These data suggested that while both students seemed to understand the university's plagiarism policy, their texts displayed patchwriting and inappropriate citation which the authors discuss in relation to the complexity of sources in introductory courses and the difficulties of attribution for novice writers.

\section{Research choices and research methodologies}

One of the main considerations of research methodology is to ensure that the study will answer the questions it has set itself, providing a credible explanation or characterization of the issue. To achieve this, many writing researchers combine several methods, both quantitative and qualitative, to gain a fuller picture of what is always a complex reality. In fact, the concept of triangulation, the use of multiple sources of data or analytical methods, can bring greater plausibility to the interpretation of results. It obviously makes sense to view research pragmatically, adopting whatever tools seem most effective, mixing methods to increase the validity of the eventual findings. Thus, text analysts, for example, often supplement studies of writing by interviewing writers and readers to better understand how these texts are typically produced and received. It is the combination of methods which help make explicit the tacit knowledge or strategies that writers and readers bring to acts of composing or assessing writing.

However, while I have discussed methods as a list of discrete instruments, they are not an open set of options in free combination. Research of any kind begins with some major decisions, concerning the 
degree of intervention, quantification, replication, objectivity and generalization which is thought desirable or even possible. An important feature of writing research, for example, is that it tends to favour data gathered in naturalistic rather than controlled conditions. This is not to say that methods that elicit data through questionnaires, structured interviews or experiments are not employed or that they have nothing to tell us about writing. It is simply that there has been a strong preference for collecting data in authentic circumstances not specifically set up for the research, such as via classroom observations or analyses of naturally occurring texts.

Most importantly, our choice of methods are influenced by our preconceptions: our personal view of what writing is and how it might best be understood. Methods are inseparable from methodologies and methodologies are underpinned by philosophical assumptions about the nature of the world and how we can know it. The selection of methods must therefore be made with regard to a range of personal and contextual factors, as shown in Table 3.

Table 3: Factors influencing choice of methods in writing research

What are you going to study (eg writer, reader, community, text, genre, context, modality)

What stance will you take (eg disinterested, interested, participatory, interventionist)

What theoretical framework will you use (eg linguistic, cognitive, sociocultural, critical, literary)

What data will you collect? (texts, corpora, interviews, diary entries, think aloud protocols)

How we you collect the data (eg observation, elicitation, experimental, text compilation)

How we you analyze it (eg statistical, interpretive, stylistic)

How will you present it (eg report, conference presentation, research article, blog)

If we try and isolate methods from this complex, to see data collection instruments as something separate from how we see our role as researchers and our view of writing, then we remove them from the assumptions which influence our research and inform our interpretations. In other words, focusing on methods alone disguises their epistemological aspects and sees them only as tools for collecting and analyzing data rather than ways of testing our preconceptions. In sum, our views about writing influence the methods we select while our methods reinforce our views about writing. 


\section{Writing paradigms and research methods}

Language research methodologies can be compared across a number of dimensions. Most obviously, we often see quantitative research, which involves statistics and manipulating numerical data, being contrasted with qualitative research, which is more holistic and process-oriented. The questions raised in Table 3, moreover, imply other divergences, such as theoretical vs applied, objective vs subjective, experimental vs ethnographic, psychometric vs naturalistic and system vs instance. Each of these contrasts can help us interrogate different methods and evaluate their value in particular contexts. When considering writing research, we can fold these polar distinctions into a number of predominant paradigms. These are six broad ways of viewing writing to which researchers often orient and which lead them to value some methods over others. These are summarized in Table 4 and discussed below. Table 4: Perspectives on writing and research methods

\begin{tabular}{llll} 
Perspective & View of writing & Main methodologies & Research methods \\
expressive activity & writing is a creative act & ethnography, case study & elicitation, observation \\
cognitive activity & writing is a thinking process & experiment, ethnography & $\begin{array}{l}\text { Observation, } \\
\text { introspection, elicitation }\end{array}$ \\
situated activity & writing is contextual performance & ethnography, & observation, introspection \\
& & auto-ethnography & elicitation, text analysis \\
completed activity & texts are rule-structured objects & text analysis & text analysis \\
social activity & texts express community purposes & discourse analysis, case study & text analysis, elicitation \\
ideology & texts reinforce power relations & critical analysis, case study & text analysis, observation \\
\hline
\end{tabular}

\section{Writing as expressive activity}

The Expressivist view rejects a definition of writing based on correct grammar and usage and sees it as a creative act of discovery in which the process is as important as the product to the writer. Writing is learnt and not taught; it is an act of imagination and self-discovery (Park, 2005). This idea, however, is challenging to L2 writers from cultures which place less emphasis on individualism. By videotaping peer review sessions and interviewing participants about their reluctance to critique their peers' essays, for example, Carson and Nelson (1996) found that PRC and Taiwanese students not only sought to preserve group harmony, but felt they lacked the individual authority to comment on others' writing. 


\section{Writing as cognitive activity}

For many researchers, writing is a cognitive performance which can be modelled by analogy with computer processing. Essentially writing is seen as a problem-solving activity: how writers approach a writing task as a problem and bring intellectual resources to solving it. It is through writing that individuals discover and formulate their ideas. A great deal of research has revealed the complexity of planning and editing activities, the influence of different writing tasks and the value of examining what writers do through a series of writing drafts. Observation and self-report are widely used methods in this paradigm. In particular, these studies have made considerable use of writers' verbal reports while composing (eg. Manchon, et al,2005), keystroke logging of writers (Van Waes , 2009), task observation (e.g. Bosher, 1998), and retrospective interviews (eg. Ferris et al, 2013).

\section{Writing as completed activity}

Once the main approach to the study of writing, there is still an active research agenda which sees texts as a product or an artefact of activity independent of particular contexts and outside the personal experiences of writers and readers. Researchers follow Saussure's (1986) famous binary and see texts as langue, or language as an abstract system which pre-exists users, rather than parole or instances of communication. They therefore tend to count features and infer rules of usage. Once this involved enumerating the T-unit length of a text to determine the overall syntactic complexity of language samples or measure the maturity of learners' writing. More recently, large corpora have been used to identify how features such as evaluative adjectives like nice, good and great are typically used (De Cock, 2011) or to assess improvement in student essays by measuring increases in morphemes, words and clauses (White, 2007). Alternatively, learner corpora can be studied to see the effect of L1 transfer (e.g. Nesselhauf, 2005). Such research describes language rather than writing, neglecting the fact that all texts include what writers suppose their readers will know, and how they will use the text. Thus no text can be fully explicit or universally 'appropriate' so such analyses are limited to a static product not the result of a writer's dynamic effort to make meaning. 


\section{Writing as situated activity}

For others, writing is a situated activity, placing emphasis on the physical and experiential contexts in which writing occurs - what Nystrand (1987) calls the 'situation of expression'. This view sees writing as influenced both by the personal attitudes and prior experiences that the writer brings to writing and the impact of the specific political and institutional contexts in which it takes place. Prior puts it like this:

When seen as situated activity, writing does not stand alone as the discrete act of a writer, but emerges as a confluence of many streams of activity: reading, talking, observing, acting, making, thinking and feeling as well as transcribing words on

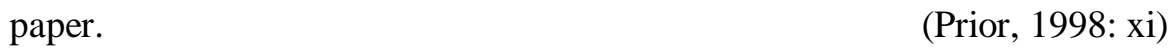

(Prior, 1998: xi)

Ethnographic approaches are often used in an attempt to give an holistic explanation of behaviour using a variety of methods and drawing on the understandings of insiders themselves to minimize the assumptions brought to the event by the researcher (Ramanathan \& Atkinson, 1999).

By using detailed observations of acts of writing, participant interviews, analyses of surrounding practices and other techniques, researchers have developed accounts of local writing contexts. These descriptions give significant attention to the experiences of writers and their understandings of the immediate context as they write. An example is Hyland's (1998) investigation of six ESL writers' reactions to, and uses of, teacher written feedback at a New Zealand university. She used a longitudinal approach which drew on a variety of data sources including observation notes, interview transcripts, think aloud protocols and written texts. However, concentrating on the local setting can fail to capture the culture and event within which the action is embedded and which their writing must invoke. Texts do not function communicatively at the time they are composed but when they are read, as they anticipate particular readers and the responses of those readers to what is written. Texts evoke a social milieu which intrudes upon the writer and activates specific responses to recurring tasks.

\section{Writing as social activity}

Once again, this perspective understands writing as a noun rather than as verb, but the focus shifts from the formal features of isolated texts toward the whole text as an instance of language functioning 
in a context of human activity. The emphasis is on discourse rather than texts as objects (Author, 2016), so here the linguistic patterns of texts point to contexts beyond the page or screen, implying a range of social constraints and choices which operate on writers in any context. Here the writer is neither a creator working through a set of cognitive processes nor an interactant engaging with a reader, but a member of a socially and rhetorically constituted community. The writer has certain goals and intentions, certain relationships to his or her readers, and certain information to convey, and the forms of a text are resources used to accomplish these. A variety of approaches have considered texts as discourse, but all have tried to discover how writers organize language to produce coherent, purposeful prose for particular groups of readers.

\section{Writing as ideology}

A final perspective on writing also emphasises the importance of social context but stresses that the key dimension of a context are the relations of power that exist in it and the ideologies that maintain these relations. The importance of power as mediating discourse and social groups has been explored most extensively by researchers working in Critical Discourse Analysis (CDA) which attempts "to unpack the ideological underpinnings of discourse that have become so naturalized over time that we begin to treat them as common, acceptable and natural features of discourse" (Teo 2006). So while this approach typically involves analyses of texts, it also considers their relationship to the wider social environment and the part they play for individuals within specific situations.

Essentially, ideology is concerned with how indviduals experience the world and how these experiences are reproduced through their writing. Fairclough (1992) uses the term 'orders of discourse' to refer to the relatively stable configurations of discourse practices found in particular domains or institutions. These are frames for interaction such as lab reports, newspaper editorials, student records, academic articles, and so on, which have prestige value in different institutions and which are ideologically shaped by its dominant groups. They provide writers with templates for appropriate ways of writing, which means that any act of writing, or of teaching writing, is embedded in ideological assumptions. While CDA does not subscribe to any single method, some researchers 
draw on discourse analysis informed by Systemic Functional Linguistics (Young \& Harrison, 2004). This is because the model sees language as a system of linguistic features which offer choices to users, but which are circumscribed in situations of unequal power. SFL thus offers CDA a way of analysing the relations between language and social contexts, making it possible to ground concerns of power and ideology in the details of discourse.

\section{Conclusions}

Two obvious points to mention in closing are that 1) there is no perfect research design or research method as the tools a researcher selects will depend on the purpose and context of the study; and that 2) methods are inseparable from theories and how we understand writing. We may select methods in order to understand writing but at the same time we select them because of the way we understand writing. Our assumptions drive our interests and our research choices so that we cannot say that any method is better than any other in unpacking the intricacies of writing and telling us what it 'really is'. Different methods and methodologies have been developed to do certain things and they differ in their capacity to do the same things. They not only answer different questions, but also give us different answers to the same questions. It makes no sense, in other words, to say what writing is independently of a theory.

The connections I have suggested between writing paradigms and inquiry methods encourage us to spell out our views about writing and about our role in the research process. This not only helps us think about our project more clearly, but is also a valuable professional development exercise in itself, sharpening our perceptions and clarifying our thinking. What questions are worth asking? What counts as appropriate data? Should it be collected naturalistically or in controlled conditions? What role will statistics play in interpreting it? How generalizable and replicable should the study be? These questions are asked by all those undertaking research and depend on what they understand writing to be and how it can be known. Clearly the complexity of writing requires multiple methods, viewing a writer, a context or set of texts though multiple lenses. But we need to be aware of the different ways of knowing that underpin our choices and make these explicit in our interpretations. 
The bottom line is that perspectives don't determine methods, but they do influence how we approach research, particularly the things we think it might be interesting to study, the kinds of questions we ask about them, and the data we need to answer those questions.

\section{References}

Author. (2013).

Author. (2016).

Author \& Other (2016).

Biber, D., Conrad, S. \& Cortes, V. (2004). If you look at...: Lexical bundles in university teaching and textbooks. Applied linguistics. 25L 371-405.

Bosher, S. (1998) The composing processes of three southeast Asian writers at the post-secondary level: an exploratory study. Journal of Second Language Writing, 7(2): 205-33.

Brown, R. (2011). Manuscript makeovers: A Rhetorical Study of Revisions Made to Dissertations Turned into Books. Journal of Scholarly Publishing. 42(4), 442475

Burns A, 2013, 'Innovation through action research and teacher-initiated change', in Hyland K; Wong LCL (ed.) Innovation and Change in English Language Education, Routledge, Oxon, pp. 90 - 105

Carson, J., \& Nelson, G. (1996). Chinese students' perceptions of ESL peer response group interaction. Journal of Second Language Writing, 5, 1-19.

Chamcharatsri, P (2009). Negotiating Identity from Auto-ethnography: Second Language Writers' Perspectives. Asian EFL Journal. Professional Teaching Articles. 38: 3-19

De Cock, S. (2011) Preferred patterns of use of positive and negative evaluative adjectives in native and learner speech: an ELT perspective. In Frankenberg-Garcia et al (eds.). New trends in corpora and language learning. London: Continuum pp 198-212.

Evans, S. and Green, C. (2007). Why EAP is necessary: A survey of Hong Kong tertiary students. Journal of English for Academic Purposes, 6, 1: 3-17

Fairclough, N. (1992) Discourse and social change. Cambridge: Polity Press. 
Ferris, D., Liu, H., Sinha, A \& Senna, M. (2013). Written corrective feedback for individual L2 writers. Journal of Second Language Writing 22 : 307-329

Hesse-Biber, S. \& Leavy, P. (2006). The practice of qualitative research. Thousand Oaks: Sage. Hyland, F. (1998). The impact of teacher written feedback on individual writers. Journal of Second Language Writing, 7, 255-286.

Lei, X. (2008). Exploring a sociocultural approach to writing strategy research: Mediated actions in writing activities. Journal of Second Language Writing. 17, 4: 217-236

Leijten, M. \& Van Waes, L. (2013). Keystroke Logging in Writing Research Using Inputlog to Analyze and Visualize Writing Processes. Written Communication. 30 (3): 358-392

Li, Y-Y. (2006). “A doctoral student of Physics writing for publication; a socio-politically-oriented case study”. English for Specific Purposes 25: 456-478.

Li, YY (2007) Apprentice scholarly writing in a community of practice: An intraview of an NNES graduate student writing a research article. TESOL quarterly 41 (1): 55 -79

Li, Y. \& Casanave, C. (2012). Two first-year students' strategies for writing from sources: Patchwriting or plagiarism? Journal of Second Language Writing. 21 (2): 165-180

Liu, P-H \& Tannacito, D. (2013) Resistance by L2 writers: The role of racial and language ideology in imagined community and identity investment. Journal of Second Language Writing. 22, 4: $355-373$

Louhiala-Salminen, L. (2002) The fly's perspective: discourse in the daily routine of a business manager. English for Specific Purposes 21, 3: 211-231

Lundstrom, K. \& Baker, W. (2009). To give is better than to receive: The benefits of peer review to the reviewer's own writing. Journal of Second language Writing.

Manchon, R. M., Murphy, L. \& Roca de Larios, J. (2005). Using concurrent protocols to explore L2 writing processes: Methodological issues in the collection and analysis of data. In P. K. Matsuda \& T. Silva (Eds.), Second Language Writing Research: Perspectives on the Process of Knowledge Construction (pp. 191-205). Mahwah, NJ: Erlbaum. 
Maréchal, G. (2010). Autoethnography. In A. J. Mills, G. Durepos \& E. Wiebe (Eds.), Encyclopedia of case study research (Vol. 2, pp. 43-45). Thousand Oaks, CA: Sage Publications.

Martin, J. (2004). Mourning - How We Get Aligned. Discourse and Society, 15(2-3), 321-344.

McGowan, H. (2011) Planning a Comparative Experiment in Educational Settings. Journal of Statistics Education 19, 2. www.amstat.org/publications/jse/v19n2/mcgowan.pdf

Nesselhauf, N. (2005). Collocations in a learner corpus. Amsterdam: John Benjamins.

Norris, J. \& Ortega, L. (2006) Effectiveness of L2 instruction: a research synthesis and quantitative meta-analysis. Language Learning 50: 417-528.

Nystrand, M. (1987) The role of context in written communication. In R. Horowitz, and S.J. Samuels (eds), Comprehending oral and written language (pp. 197-214). San Diego, CA: Academic Press.

Paltridge, B. (2008). Textographies and the researching and teaching of writing. Iberica, 15, 9-24.

Park, K. (2005). Writing at the edge: narrative and writing process. Bern: Peter Lang.

Ramanathan, V. and Atkinson, D. (1999) Ethnographic approaches and methods in L2 writing research: a critical guide and review. Applied Linguistics, 20(1): 44-70.

Stake, R. (1995). The art of case study research. Thousand Oaks, CA: Sage.

Starfield, S., Paltridge, B., Ravelli, L. (2014). Researching academic writing: What textography affords. In J. Huisman, M. Tight (Eds.), Theory and method in higher education research II, (pp. 103-120). Oxford, UK: Emerald.

de Saussure, F. (1986). Course in general linguistics (3rd ed.). (R. Harris, Trans.). Chicago: Open Court Publishing.

Teo, A. K. (2006). Social-interactive writing for English language learners. The CATESOL Journal, 18, $160-178$.

Tognini-Bonelli, E. (2001). Corpus linguistics at work. Amsterdam: John Benjamins.

Truscott, J. (2007). The effect of error correction on learners' ability to write accurately. Journal of Second Language Writing, 16, 255-272.

Van Waes, L., Leijten, M., \& Van Weijen, D. (2009). Keystroke logging in writing research: Observing writing processes with Inputlog. GFL-German as a foreign language, 2(3), 41-64 
Weigle, S. (1999). Investigating Rater/Prompt Interactions in Writing Assessment: Quantitative and Qualitative Approaches. Assessing writing 6 (2): 145-178.

Wong. A. T.Y. (2005). Writers' mental representations of the intended audience and of the rhetorical purpose for writing and the strategies that they employed when they composed. System, 33: 29-47

Xiao, R., McEnery, T. \& Qian, Y. (2006) Passive constructions in English and Chinese: A corpusbased contrastive study. Languages in Contrast, 6, 1: 109-149

Yang, C., Hu, G. \& Wang, L. (2014). Reactivity of concurrent verbal reporting in second language writing. Journal of Second Language Writing. 24: 51-70.

Yin, R. (2014). Case study research: Design and methods. 5th ed. Thousand Oaks, CA: Sage

Young, L. \& Harrison, C. (2004). Systemic Functional Linguistics and Critical Discourse Analysis London: Continuum

Zhu, W \& Flaitz, J. (2005) Using Focus Group Methodology to Understand International Students' Academic Language Needs: A Comparison of Perspectives. TESOL-EJ. 8 (4) A-3. 